\title{
The Prism tableau model for Schubert polynomials
}

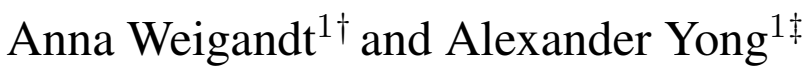 \\ ${ }^{1}$ Dept. of Mathematics, U. Illinois at Urbana-Champaign, Urbana, IL 61801, USA
}

\begin{abstract}
The Schubert polynomials lift the Schur basis of symmetric polynomials into a basis for $\mathbb{Z}\left[x_{1}, x_{2}, \ldots\right]$. We suggest the prism tableau model for these polynomials. A novel aspect of this alternative to earlier results is that it directly invokes semistandard tableaux; it does so as part of a colored tableau amalgam. In the Grassmannian case, a prism tableau with colors ignored is a semistandard Young tableau. Our arguments are developed from the Gröbner geometry of matrix Schubert varieties.

Résumé. Les polynômes de Schubert prolongent la base des polynômes de Schur des polynômes symétriques en une base de $\mathbb{Z}\left[x_{1}, x_{2}, \ldots\right]$. Nous suggérons le modèle de tableaux de prismes pour ces polynômes. Un nouvel aspect de cette alternative aux résultats antérieurs est qu'elle utilise directement les tableaux semi-standards; Ces derniers apparaissent sous forme d'amalgames de tableaux colorés. Dans le cas Grassmannien, un tableau de prisme avec les couleurs omises est un tableau de Young semi-standard. Nos arguments sont basés sur la géométrie de Gröbner des variétés de matrices de Schubert.
\end{abstract}

Keywords. Schubert polynomials, Gröbner geometry, Young tableau

\section{Introduction}

\subsection{Overview}

A. Lascoux-M.-P. Schützenberger [LaSh82a] recursively defined an integral basis of Pol $=\mathbb{Z}\left[x_{1}, x_{2}, \ldots\right]$ given by the Schubert polynomials $\left\{\mathfrak{S}_{w}: w \in S_{\infty}\right\}$. If $w_{0}$ is the longest length permutation in the symmetric group $S_{n}$ then $\mathfrak{S}_{w_{0}}:=x_{1}^{n-1} x_{2}^{n-2} \cdots x_{n-1}$. Otherwise, $w \neq w_{0}$ and there exists $i$ such that $w(i)<w(i+1)$. Now one sets $\mathfrak{S}_{w}=\partial_{i} \mathfrak{S}_{w s_{i}}$, where $\partial_{i} f:=\frac{f-s_{i} f}{x_{i}-x_{i+1}}$ (since the polynomial operators $\partial_{i}$ form a representation of $S_{n}$, this definition is self-consistent.) It is true that under the standard inclusion $\iota: S_{n} \hookrightarrow S_{n+1}, \mathfrak{S}_{w}=\mathfrak{S}_{\iota(w)}$. Thus one can refer to $\mathfrak{S}_{w}$ for each $w \in S_{\infty}=\bigcup_{n \geq 1} S_{n}$.

Textbook understanding of the ring Sym of symmetric polynomials centers around the basis of Schur polynomials and its successful companion, the theory of Young tableaux. Since Schur polynomials are instances of Schubert polynomials, the latter basis naturally lifts the Schur basis into Pol. Yet, it is also true that Schubert polynomials have nonnegative integer coefficients. Consequently, one has a natural problem:

\footnotetext{
†Email: weigndt2@uiuc.edu.

${ }_{\ddagger}^{\ddagger}$ Email: ayong@uiuc. edu. AY was supported by an NSF grant. 
Is there a combinatorial model for Schubert polynomials that is analogous to the semistandard tableau model for Schur polynomials?

Indeed, multiple solutions have been discovered over the years, e.g., [Ko90], [BiJoSt93], [BeBi93], [FoSt94], [FoKi96], [FoGrReSh97], [Ma98], [BeSo98, BeSo02], [BuKrTaYo04] and [CoTa13] (see also [LaSh85]). In turn, the solutions [BiJoSt93, BeBi93, FoSt94, FoKi96] have been the foundation for a vast literature at the confluence of combinatorics, representation theory and combinatorial algebraic geometry.

We wish to put forward another solution - a novel aspect of which is that it directly invokes semistandard tableaux. Both the statement and proof of our alternative model build upon ideas about the Gröbner geometry of matrix Schubert varieties $X_{w}$. We use the Gröbner degeneration of $X_{w}$ and the interpretation of $\mathfrak{S}_{w}$ as mutidegrees of $X_{w}$ [KnMi05]. Actually, a major purpose of loc. cit. is to establish the geometric naturality of the combinatorics of [BiJoSt93, BeBi93, FoKi96]. Our point of departure is stimulated by later work of A. Knutson on Frobenius splitting [Kn09, Theorem 6 and Section 7.2].

\subsection{The main result}

We recall some permutation combinatorics found in, e.g., in [Ma01]. The diagram of $w$ is $D(w)=$ $\left\{(i, j): 1 \leq i, j \leq n, w(i)>j\right.$ and $\left.w^{-1}(j)>i\right\} \subset n \times n$. Let $\mathcal{E} s s(w) \subset D(w)$ be the essential set of $w$ : the southeast-most boxes of each connected component of $w$. The rank function is $r_{w}(i, j)=\#\{t \leq$ $i: w(t) \leq j\}$.

Define $w$ to be Grassmannian if it has at most one descent, i.e., at most one index $k$ such that $w(k)>$ $w(k+1)$. If in addition $w^{-1}$ is Grassmannian then $w$ is biGrassmannian. For $e=(i, j) \in \mathcal{E} s s(w)$ let $R_{e}$ be the $\left(i-r_{w}(i, j)\right) \times\left(j-r_{w}(i, j)\right)$ rectangle with southwest corner at position $(i, 1)$ of $n \times n$. The shape of $w$ is $\lambda(w)=\bigcup_{e \in \mathcal{E} s(w)} R_{e}$ :

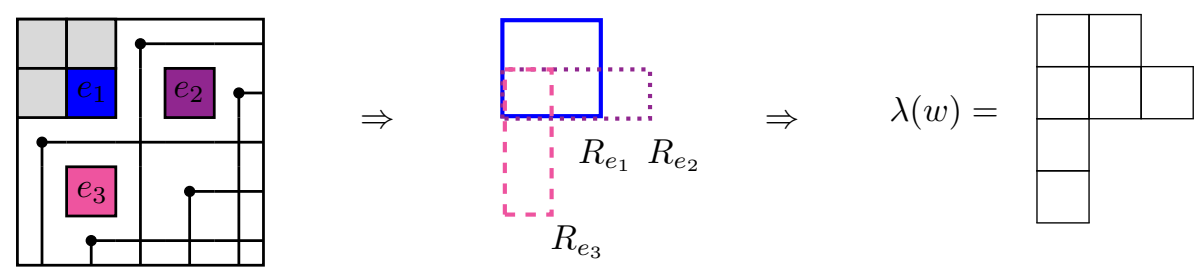

Fig. 1: The diagram of $w=35142$ (with color coded essential set $\left\{e_{1}, e_{2}, e_{3}\right\}$ ), the overlay of $R_{e_{1}}, R_{e_{2}}, R_{e_{3}}$, and the shape $\lambda(w)$.

A prism tableau $T$ for $w$ fills $\lambda(w)$ with colored labels (one color for each $e \in \mathcal{E} s s(w)$ ) such that the labels of color $e$ :

(S1) sit in a box of $R_{e}$;

(S2) weakly decrease along rows from left to right;

(S3) strictly increase along columns from top to bottom; and

(S4) are flagged: a label is no bigger than the row of the box it sits in. 
Let $d_{i}(w)$ be the number of distinct values (ignoring color) seen on the $i$-th antidiagonal (i.e., the one meeting $(i, 1)$ ), for $i=1,2, \ldots, n$. We say $T$ is minimal if $\sum_{i=1}^{n} d_{i}(w)=\ell(w)$, where $\ell(w)$ is the Coxeter length of $w$.

Let $\ell_{c}$ be a label $\ell$ of color $c$. Labels $\left\{\ell_{c}, \ell_{d}, \ell_{e}^{\prime}\right\}$ in the same antidiagonal form an unstable triple if $\ell<\ell^{\prime}$ and replacing the $\ell_{c}$ with $\ell_{c}^{\prime}$ gives a prism tableau. See Example 1.3

Let $\operatorname{Prism}(w)$ be the set of minimal prism tableaux with no unstable triples. Finally, set

$$
\mathfrak{P}_{w}\left(x_{1}, \ldots, x_{n}\right):=\sum_{T \in \operatorname{Prism}(w)} \operatorname{wt}(T), \text { where } \mathrm{wt}(T)=\prod_{i} x_{i}^{\# \text { of antidiagonals containing } i} .
$$

Theorem 1.1. $\mathfrak{S}_{w}\left(x_{1}, \ldots, x_{n}\right)=\mathfrak{P}_{w}\left(x_{1}, \ldots, x_{n}\right)$.

Example 1.2 (Reduction to semistandard tableaux). Consider the Grassmannian permutation $w=246135$. Conflating prism tableaux with their weights, Theorem 1.1 asserts:

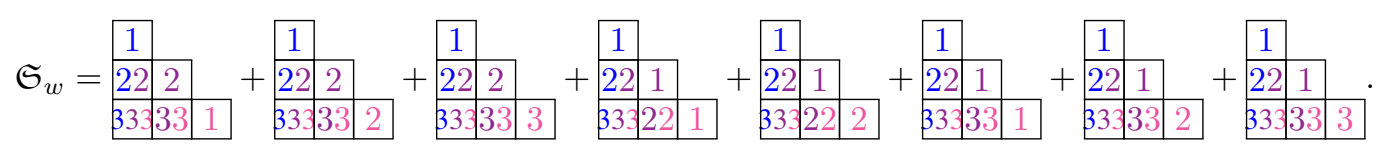

Forgetting colors gives the following expansion of the Schur polynomial:

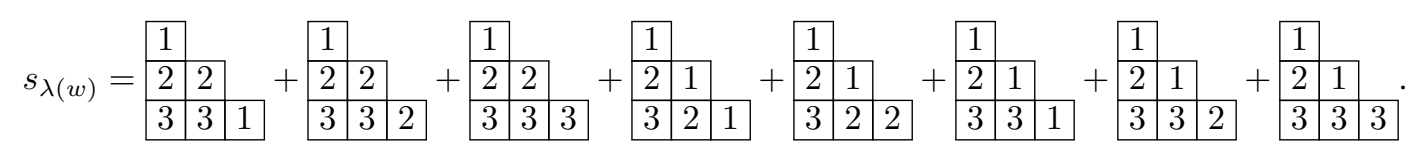

In general, if $w$ is Grassmannian then $\lambda(w)$ is a (French) Young diagram. Moreover, each cell of $T \in \operatorname{Prism}(w)$ uses only one number. Replacing each set in $T$ by the common value gives a reverse semistandard tableau. Thus $\mathfrak{P}_{w}=s_{\lambda}(w)$ follows.

Prism tableaux provide a means to understand the $R C$-graphs of [BeBi93, FoKi96]. We think of the $\# \mathcal{E} s s(w)$-many semistandard tableaux of a prism tableau $T$ as the "dispersion" of the associated $R C$ graph through T. See Sections 4.1 and 4.3.

Minimality and the unstable triple condition bond the tableau of each color, which is one reason why we prefer not to think of a prism tableau as merely a $\# \mathcal{E} s s(w)$-tuple:

\begin{tabular}{|c|c|c|c|c|}
\hline$T$ & \begin{tabular}{|l|l|l|}
11 & 1 & 1 \\
22 & 1 & \multicolumn{1}{|c}{} \\
\cline { 1 - 2 } 33 & 3 & \\
\cline { 1 - 2 }
\end{tabular} & \begin{tabular}{|l|l|l|}
11 & 1 & 1 \\
21 & 1 & \multicolumn{1}{|}{} \\
\cline { 1 - 1 } 33 & 3 & \\
\cline { 1 - 2 }
\end{tabular} & \begin{tabular}{|l|l|l|}
11 & 1 & 1 \\
22 & 1 & \multicolumn{1}{|l}{} \\
\cline { 1 - 2 } 33 & 2 & \\
\cline { 1 - 2 }
\end{tabular} & \begin{tabular}{|l|l|l|}
11 & 1 & 1 \\
21 & 1 & \multicolumn{1}{|}{} \\
\cline { 1 - 2 } 33 & 2 & \\
\end{tabular} \\
\hline $\operatorname{wt}(T)$ & $x_{1}^{3} x_{2} x_{3}^{2}$ & $x_{1}^{3} x_{2} x_{3}^{2}$ & $x_{1}^{3} x_{2}^{2} x_{3}$ & $x_{1}^{3} x_{2}^{2} x_{3}$ \\
\hline
\end{tabular}

Example 1.3 (Unstable triples). Let $w=42513$. Then $\# \mathcal{E} s s(w)=3$. The minimal prism tableaux and their weights are:

The second and the fourth tableaux have an unstably paired label. In both tableaux, the pink 1 in the second antidiagonal is replaceable by a pink 2 . So $\mathfrak{S}_{w}=x_{1}^{3} x_{2} x_{3}^{2}+x_{1}^{3} x_{2}^{2} x_{3}$. 


\section{Main idea of the model and its proof}

Let $\mathrm{G}=\mathrm{GL}_{n}$ and $\mathrm{B}$ and $\mathrm{B}^{+}$the Borel subgroups of lower and upper triangular matrices in $\mathrm{G}$. Identify the flag variety with the coset space $B \backslash G$. Let $T$ be the maximal torus in $B$. Suppose $\mathfrak{X} \subset B \backslash G$ is an arbitrary subvariety and $\pi: G \rightarrow B \backslash G$ is the natural projection. Then

$$
X=\overline{\pi^{-1}(\mathfrak{X})} \subseteq \operatorname{Mat}_{n \times n}
$$

carries a left $\mathrm{B}$ action and thus the action of $\mathrm{T}$. Therefore, one can speak of the equivariant cohomology class

$$
[X]_{T} \in H_{\mathrm{\top}}\left(\mathrm{Mat}_{n \times n}\right) \cong \mathbb{Z}\left[x_{1}, \ldots, x_{n}\right] .
$$

Moreover, the polynomial $[X]_{\mathrm{T}}$ is a coset representative under the Borel presentation of

$$
[\mathfrak{X}] \in H^{\star}(\mathrm{B} \backslash \mathrm{G}, \mathbb{Z}) \cong \mathbb{Z}\left[x_{1}, \ldots, x_{n}\right] / I^{S_{n}},
$$

where $I^{S_{n}}$ is the ideal generated by (non-constant) elementary symmetric polynomials (cf. [KnMi05, pg. 1280]). This is a key perspective of work of A. Knutson-E. Miller [KnMi05] when $\mathfrak{X}$ is a Schubert variety.

Let $Y \subseteq$ Mat $_{n \times n}$ be an equidimensional, reduced union of coordinate subspaces. Given $\mathcal{P} \subset n \times n$, we represent $\mathcal{P}$ visually as a collection of + 's in the $n \times n$ grid. We say $\mathcal{P}$ is a plus diagram for $Y$, if

$$
\mathcal{L}_{\mathcal{P}}:=\left\{M \in \operatorname{Mat}_{n \times n}: M_{i, j}=0 \text { if }(i, j) \in \mathcal{P}\right\} \subset Y .
$$

Let Plus $(Y)$ be the set of all such plus diagrams. Let MinPlus $(Y)$ be the set of minimal plus diagrams, i.e., those $\mathcal{P}$ for which removing any + would not return an element of $\operatorname{Plus}(Y)$. We refer to the union of plus diagrams as an overlay to emphasize whenever $(i, j)$ is in $\mathcal{P}$ or $\mathcal{P}^{\prime}$, the diagram for $\mathcal{P} \cup \mathcal{P}^{\prime}$ also has a + in position $(i, j)$.

Each $\mathcal{P}$ corresponds $1: 1$ to a face of the Stanley-Reisner complex $\Delta_{Y}$. Let $\Delta_{n \times n}$ be the power set of $\{(i, j): 1 \leq i, j \leq n\}$. Then $\Delta_{Y} \subseteq \Delta_{n \times n}$ and for each $\mathcal{P}$ one has the face

$$
\mathcal{F}_{\mathcal{P}}=\{(i, j): 1 \leq i, j \leq n \text { and }(i, j) \notin \mathcal{P}\} .
$$

The faces of $\Delta_{Y}$ are ordered by reverse containment of their plus diagrams. Thus, facets (maximal dimensional faces) of $\Delta_{Y}$ coincide with elements of $\operatorname{MinPlus}(Y)$. In addition, taking the overlay of $\mathcal{P} \in \operatorname{Plus}(Y)$ and $\mathcal{Q} \in \operatorname{Plus}(Z)$ corresponds to intersecting faces in the Stanley-Reisner complex:

$$
\mathcal{F}_{\mathcal{P} \cup \mathcal{Q}}=\mathcal{F}_{\mathcal{P}} \cap \mathcal{F}_{\mathcal{Q}} \in \Delta_{Y} \cap \Delta_{Z} .
$$

Through the interpretation of $[Y]_{T}$ as a multidegree, one may express $[Y]_{T}$ as a generating series over $\operatorname{MinPlus}(Y)$. That is,

$$
[Y]_{T}=\sum_{\mathcal{P} \in \operatorname{MinPlus}(Y)} \text { wt }(\mathcal{P}), \quad \text { where } \mathrm{wt}(\mathcal{P})=\prod_{i=1}^{n} x_{i}^{\# \text { of }+ \text { 's in row } i} .
$$

For details, the reader may consult [MiSt05]; see Chapter 1 and Chapter 8 (and its notes).

Suppose $\prec$ is any term order on $\mathbb{C}\left[\mathrm{Mat}_{n \times n}\right]$ and $X^{\prime}:=$ init $_{\prec} X$. Since $X$ is T-stable the same is true of $X^{\prime}$; thus $\left[X^{\prime}\right]_{\mathrm{T}}$ is defined. Gröbner degeneration preserves the T-equivariant class, so $[X]_{\mathrm{T}}=\left[X^{\prime}\right]_{\mathrm{T}}$. 
Suppose $X^{\prime}$ is reduced, and hence a reduced union of coordinate subspaces. Since $\mathfrak{X}$ was assumed to be irreducible, then $X$ is irreducible. So by [KaSt95, Theorem 1] the Stanley-Reisner complex $\Delta_{X^{\prime}}$ of $X^{\prime}$ is equidimensional. Hence we may apply the discussion above using $Y=X^{\prime}$ to compute $\left[X^{\prime}\right]_{\mathrm{T}}=[X]_{\mathrm{T}}$.

We are interested in understanding $\Delta_{X^{\prime}}$ under certain hypotheses on $X$. Assume that we have a collection of varieties $X, X_{1}, \ldots, X_{m} \subseteq V \cong \mathbb{C}^{N}$ such that

$$
X=X_{1} \cap X_{2} \cap \cdots \cap X_{k} .
$$

Assume $\prec$ is a term order on $\mathbb{C}[V]$ that defines a Gröbner degeneration of these varieties so that each Gröbner limit

$$
X^{\prime}:=\text { init }_{\prec} X, X_{i}^{\prime}:=\text { init }_{\prec} X_{i} \text { (for } i=1,2, \ldots, k \text { ) is reduced. }
$$

Finally, suppose

$$
X^{\prime}=X_{1}^{\prime} \cap X_{2}^{\prime} \cap \cdots \cap X_{k}^{\prime}
$$

Call $\left\{X_{i}\right\}$ a $\prec$-spectrum for $X$.

To construct a cheap example, pick any Gröbner basis $G=\left\{g_{1}, \ldots, g_{M}\right\}$ with square-free lead terms to define $X$. Now partition $G=G_{1} \cup G_{2} \cup \cdots \cup G_{s}$ and set $X_{k}$ to be cut out by $G_{k}$. On the other hand, a motivating example is A. Knutson [Kn09, Theorem 6]: given a term order $\prec$ (satisfying a hypothesis), there is a stratification of $V$ into a poset of varieties (ordered by inclusion) with the additional feature that each stratum $X$ admits a $\prec$-spectrum using higher strata.

How can a $\prec$-spectrum be used to understand the combinatorics of $\left[X^{\prime}\right]_{\mathrm{T}}$ ? Here is a simple observation:

Lemma 2.1. Let $\left\{X_{i}\right\}$ be $a \prec$-spectrum for $X$. Then

(I) $\operatorname{Plus}\left(X^{\prime}\right)=\left\{\mathcal{P}_{1} \cup \cdots \cup \mathcal{P}_{k}: \mathcal{P}_{i} \in \operatorname{Plus}\left(X_{i}^{\prime}\right)\right\}$.

(II) $\operatorname{MinPlus}\left(X^{\prime}\right) \subseteq\left\{\mathcal{P}_{1} \cup \cdots \cup \mathcal{P}_{k}: \mathcal{P}_{i} \in \operatorname{MinPlus}\left(X_{i}^{\prime}\right)\right\}$

Proof. (I): Let $\mathcal{P} \in \operatorname{Plus}\left(X^{\prime}\right)$. Then $\mathcal{L}_{\mathcal{P}} \subseteq X^{\prime} \subseteq X_{i}^{\prime}$ for all $i$. Therefore $\mathcal{P} \in \operatorname{Plus}\left(X_{i}^{\prime}\right)$ and trivially $\mathcal{P}=\mathcal{P} \cup \ldots \cup \mathcal{P}$, proving " $\subseteq$ ". For the other containment, suppose $\mathcal{P}_{i} \in \operatorname{Plus}\left(X_{i}^{\prime}\right)$ for $1 \leq i \leq k$ and let $\mathcal{P}=\mathcal{P}_{1} \cup \cdots \cup \mathcal{P}_{k}$. Then $\mathcal{L}_{\mathcal{P}}=\mathcal{L}_{\mathcal{P}_{1}} \cap \ldots \cap \mathcal{L}_{\mathcal{P}_{k}}$ and hence $\mathcal{L}_{\mathcal{P}} \subseteq \mathcal{L}_{\mathcal{P}_{i}} \subseteq X_{i}^{\prime}$. So $\mathcal{P} \in \operatorname{Plus}\left(X_{i}^{\prime}\right)$ for each $i$, which implies $\mathcal{P} \in \operatorname{Plus}\left(X^{\prime}\right)$.

(II): Let $\mathcal{P} \in \operatorname{MinPlus}\left(X^{\prime}\right)$. By (I), $\mathcal{P} \in \operatorname{Plus}\left(X_{i}^{\prime}\right)$ for each $i$. Then there exists $\mathcal{P}_{i} \in \operatorname{MinPlus}\left(X_{i}^{\prime}\right)$ so that $\mathcal{P}_{i} \subseteq \mathcal{P}$. Then $\mathcal{P} \supseteq \mathcal{P}_{1} \cup \cdots \cup \mathcal{P}_{k} \in \operatorname{Plus}\left(X^{\prime}\right)$ by (I). As $\mathcal{P}$ is minimal, this is an equality.

Our point is that in good cases, the plus diagrams of $X_{i}^{\prime}$ are "simpler" to understand than those of $X$. Lemma 2.1 (II) says that one can think of each $\mathcal{P} \in \operatorname{MinPlus}(X)$ as an overlay $\mathcal{P}=\mathcal{P}_{1} \cup \cdots \cup \mathcal{P}_{k}$ of these simpler $\mathcal{P}_{i}$. Of course, this representation is not unique in general, so one can make a choice of representation for each $\mathcal{P}$. The hope is to transfer understanding of the combinatorics of $\operatorname{MinPlus}\left(X_{i}\right)$ to the combinatorics of $\operatorname{MinPlus}(X)$.

\section{Proof of the Theorem 1.1}

We now carry out the ideas described in Section 2 in the case of Schubert varieties. 


\subsection{Matrix Schubert varieties and Schubert polynomials}

The flag variety $\mathrm{B} \backslash \mathrm{G}$ decomposes into Schubert cells $\mathfrak{X}_{w}^{\circ}:=\mathrm{B} \backslash \mathrm{B} w \mathrm{~B}^{+}$indexed by $w \in S_{n}$. The Schubert variety is the Zariski-closure $\mathfrak{X}_{w}:=\overline{\mathfrak{X}}_{w}^{\circ}$. The matrix Schubert variety is

$$
X_{w}:=\overline{\pi^{-1}\left(\mathfrak{X}_{w}\right)} \subset \operatorname{Mat}_{n \times n} .
$$

Let $Z=\left(z_{i j}\right)_{1 \leq i, j \leq n}$ be the generic $n \times n$ matrix. The Schubert determinantal ideal is

$$
I_{w}=\left\langle r_{w}(i, j)+1 \text { minors of the the northwest } i \times j \text { submatrix of } Z\right\rangle \subset \mathbb{C}\left[\operatorname{Mat}_{n \times n}\right] .
$$

In [Fu91, Lemma 3.10] it is proved that $I_{w}$ cuts out $X_{w}$ scheme-theoretically. Moreover in loc. cit. it is shown that $I_{w}$ is generated by the smaller set of generators coming from those $(i, j) \in \mathcal{E} s s(w)$.

By [KnMi05, Theorem A],

$$
\left[X_{w}\right]_{\mathrm{T}}=\mathfrak{S}_{w}\left(x_{1}, \ldots, x_{n}\right) \in H_{\mathrm{T}}\left(\operatorname{Mat}_{n \times n}\right) .
$$

Moreover, let $\prec_{\text {anti }}$ be any antidiagonal term order on $\mathbb{C}\left[\mathrm{Mat}_{n \times n}\right]$, i.e., one that picks off the antidiagonal term of any minor of $Z$. In [KnMi05, Theorem B] it is shown that MinPlus $\left(X_{w}^{\prime}\right)$ are in a transparent bijection with the $R C$-graphs of [BeBi93] (cf. [FoKi96]).

For each $e \in \mathcal{E} s s(w)$, there is a unique biGrassmannian permutation $u_{e}$ such that $r_{u_{e}}(e)=r_{w}(e)$ and $\mathcal{E} s s\left(u_{e}\right)=\{e\}[$ [LaSh96]. Let

$$
\operatorname{biGrass}(w):=\left\{u_{e}: e \in \mathcal{E} s s(w)\right\}=\left\{u_{1}, \ldots, u_{k}\right\} .
$$

Call $\left\{X_{u_{1}}, \ldots, X_{u_{k}}\right\}$ the biGrassmannian $\prec_{\text {anti }}$-spectrum for $X_{w}$. By [Kn09, Section 7.2], $\left\{X_{u_{i}}\right\}$ indeed gives a $\prec_{\text {anti }}$-spectrum for $X_{w}$ over $\mathbb{Q}$. This result can also be readily obtained (over $\mathbb{Z}$ ) if one assumes the Gröbner basis result [KnMi05, Theorem B]. (It should be emphasized that one of the points of [Kn09, Section 7.2] is to reprove said Gröbner basis theorem more easily.)

\subsection{Multi-plus diagrams}

The technical core of our proof is to analyze the combinatorics of overlays of plus diagrams for the biGrassmannian $\prec$ anti-spectrum $\left\{X_{u_{1}}, \ldots, X_{u_{k}}\right\}$. Let

$$
\operatorname{Multi}(w)=\prod_{i=1}^{k} \operatorname{MinPlus}\left(X_{u_{i}}^{\prime}\right)
$$

be the set of multi-plus diagrams for $w$ : we represent $\left(\mathcal{P}_{1}, \ldots, \mathcal{P}_{k}\right) \in \operatorname{Multi}(w)$ as a placement of colored +'s in a single $n \times n$ grid, where $(a, b)$ has a + of color $u_{i}$ if $(a, b) \in \mathcal{P}_{i}$.

By Lemma 2.1.I), there is a map

$$
\text { supp }: \operatorname{Multi}(w) \rightarrow \operatorname{Plus}\left(X_{w}^{\prime}\right)
$$

given by $\left(\mathcal{P}_{1}, \ldots, \mathcal{P}_{k}\right) \mapsto \mathcal{P}_{1} \cup \ldots \cup \mathcal{P}_{k}$. Call $\mathcal{P}_{1} \cup \ldots \cup \mathcal{P}_{k}$ the support of $\left(\mathcal{P}_{1}, \ldots, \mathcal{P}_{k}\right)$. Central to our study is

$$
\operatorname{Multi}(\mathcal{P}):=\operatorname{supp}^{-1}(\mathcal{P}) \text {. }
$$




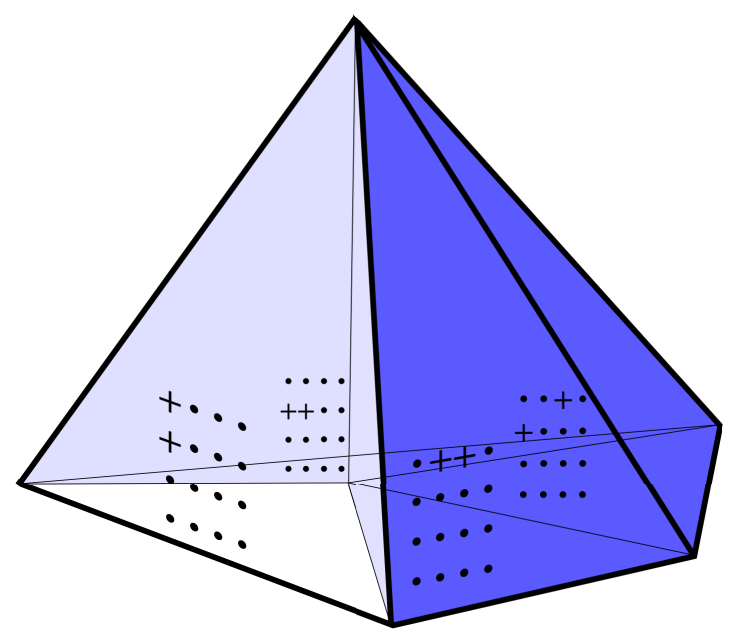

Fig. 2: The Stanley-Reisner complexes for $X_{1423}^{\prime}$ and $X_{2314}^{\prime}$ intersect to give the complex for $X_{2413}^{\prime}$. These complexes are a multicone over the depicted complex.

Example 3.1. Let $w=42513$. Then $\operatorname{biGrass}(w)=\{41235,23415,14523\}$. Now,

$$
\mathcal{P}=\left[\begin{array}{ccccc}
+ & + & + & \cdot & \cdot \\
+ & \cdot & + & \cdot & \cdot \\
+ & \cdot & \cdot & \cdot & \cdot \\
\cdot & \cdot & \cdot & \cdot & \cdot \\
\cdot & \cdot & \cdot & \cdot & \cdot
\end{array}\right] \in \operatorname{MinPlus}\left(X_{w}^{\prime}\right)
$$

One can check that

$$
\operatorname{Multi}(\mathcal{P})=\left\{\left[\begin{array}{ccccc}
++ & ++ & ++ & \cdot & \cdot \\
+ & \cdot & + & \cdot & \cdot \\
++ & \cdot & \cdot & \cdot & \cdot \\
\cdot & \cdot & \cdot & \cdot & \cdot \\
\cdot & \cdot & \cdot & \cdot & \cdot
\end{array}\right], \quad\left[\begin{array}{ccccc}
++ & + & ++ & \cdot & \cdot \\
++ & \cdot & + & \cdot & \cdot \\
++ & \cdot & \cdot & \cdot & \cdot \\
\cdot & \cdot & \cdot & \cdot & \cdot \\
\cdot & \cdot & \cdot & \cdot & \cdot
\end{array}\right]\right\}
$$

\subsection{Local moves on plus diagrams}

A southwest move is the following local operation on a plus diagram:

$$
\left[\begin{array}{cc}
\cdot & + \\
\cdot & \cdot
\end{array}\right] \mapsto\left[\begin{array}{cc}
\cdot & \cdot \\
+ & \cdot
\end{array}\right] .
$$


The inverse operation is a northeast move:

$$
\left[\begin{array}{cc}
\cdot & \cdot \\
+ & \cdot
\end{array}\right] \mapsto\left[\begin{array}{cc}
\cdot & + \\
\cdot & \cdot
\end{array}\right] .
$$

Suppose $\mathcal{E} s s(u)=\{(i, j)\}$. Define $\mathcal{D}_{\text {bot }}(u) \in \operatorname{MinPlus}\left(X_{u}^{\prime}\right)$ as the $\left(i-r_{u}(i, j)\right) \times\left(j-r_{u}(i, j)\right)$ rectangle of + 's, with southwest corner in row $i$ and column 1 . The following is well-known, and is a consequence (by specialization) of the chute and ladder moves of [BeBi93, Theorem 3.7]:

Lemma 3.2. Let $u \in S_{n}$ be biGrassmannian.

(I) MinPlus $\left(X_{u}^{\prime}\right)$ is connected and closed under the moves (3.1) and 3.2).

(II) Each $\mathcal{P} \in \operatorname{MinPlus}\left(X_{u}^{\prime}\right)$ can be obtained from $\mathcal{D}_{\text {bot }}(u)$ using only the moves 3.2.

Define a partial order on MinPlus $\left(X_{u}^{\prime}\right)$ by taking the transitive closure of the covering relation $\mathcal{P}<\mathcal{P}^{\prime}$ if $\mathcal{P}^{\prime}$ is obtained from $\mathcal{P}$ by a northeast local move $(3.2)$. Let $<^{\prime}$ be the partial order on $\operatorname{Multi}(w)$ defined as the $\mathcal{E} s s(w)$-factor Cartesian product of $<$. That is $\left(\mathcal{P}_{1}, \ldots, \mathcal{P}_{k}\right)<^{\prime}\left(\mathcal{Q}_{1}, \ldots, \mathcal{Q}_{k}\right)$ if and only if $\mathcal{P}_{i}<\mathcal{Q}_{i}$ for each $i$. Then $<^{\prime}$ induces a partial order on $\operatorname{Multi}(\mathcal{P}) \subseteq \operatorname{Multi}(w)$.

Given $\left(\mathcal{P}_{1}, \ldots, \mathcal{P}_{m}\right) \in \operatorname{Multi}(w)$, a long move is a repeated application of 3.1 (respectively, 3.2) to a single + appearing in one of the $\mathcal{P}_{i}$ 's. Recall that a lattice is a partially ordered set in which every two elements $x$ and $y$ have a least upper bound $x \vee y$ (join) and a unique greatest lower bound $x \wedge y$ (meet). It is basic that a Cartesian product of lattices is a lattice.

Theorem 3.3. Let $w \in S_{n}$ and $\mathcal{P} \in \operatorname{MinPlus}\left(X_{w}^{\prime}\right)$.

(I) $\operatorname{Multi}(\mathcal{P})$ is connected by long moves.

(II) Each (MinPlus $\left.\left(X_{u_{i}}^{\prime}\right),<\right)$ is a lattice. Consequently, $\left(\operatorname{Multi}(w),<^{\prime}\right)$ is a lattice.

(III) $\left(\operatorname{Multi}(\mathcal{P}),<^{\prime}\right)$ is a sublattice of $\left(\operatorname{Multi}(w),<^{\prime}\right)$.

To prove Theorem 3.3, we construct a path of long moves between any two $\mathcal{Q}, \mathcal{Q}^{\prime} \in \operatorname{Multi}(\mathcal{P})$, noting that this path contains the meet of $\mathcal{Q}$ and $\mathcal{Q}^{\prime}$ in $\left(\operatorname{Multi}(w),<^{\prime}\right)$, and thus is the meet for $\left(\operatorname{Multi}(\mathcal{P}),<^{\prime}\right)$. A symmetric argument produces the join. To prove the main theorem, we show there is a bijection between the set of prism tableau for $w$ and $\operatorname{Multi}(w)$. We then identify each $\mathcal{P} \in \operatorname{MinPlus}\left(X_{w}^{\prime}\right)$ with the prism tableau that corresponds to the minimum element of $\operatorname{Multi}(\mathcal{P})$ and show this is a bijection.

\section{Further discussion}

\subsection{Comparisons to the literature}

Ultimately, the evaluation of any model for Schubert polynomial rests on its success towards the Schubert problem, i.e., finding a generalized Littlewood-Richardson rule for Schubert polynomials. Due to the analogy with Sym, one hopes that a solution will not only provide merely a rule, but rather lead to an entire companion combinatorial theory. This would presumably enrich our understanding of Pol and its role in mathematics just as the Young tableau theory does for Sym.

That the prism model manifestly uses Young tableaux is our impetus for ongoing investigations that fundamental tableaux algorithms might admit prism-generalizations. 
The first rule for Schubert polynomials was conjectured by [Ko90]. This rule begins with the diagram of $w$ and evolves other subsets of $n \times n$ by a simple move, the Schubert polynomial is a generating series over these subsets. A proof is presented in [Wi99, Wi02]. Arguably, this rule is the most handy of all known rules, even though the set of Kohnert diagrams does not have a closed description.

Probably the most well-known and utilized formula is given by [BiJoSt93], which expresses the Schubert polynomial in terms of reduced decompositions of $w$. This rule is made graphical by the $R C$-graphs of [BeBi93] (cf. [FoKi96]). One can obtain any $R C$-graph for $w$ from any other by the chute and ladder moves of [BeBi93].

While neither of the above rules transparently reduces to the tableau rule for Schur polynomials, it is not too difficult to show in either case, that the objects involved do biject with semistandard tableaux, see [Ko90] and [Ko00] respectively.

We are not aware of any published bijection between the Kohnert rule and any other model for Schubert polynomials. On the other hand, there is a map between the prism tableaux and $R C$-graphs: the labels on the $i$-th antidiagonal indicate the row position of the +'s on the same antidiagonal in the associated $R C$-graph. We treat each $R C$-graph as a specific overlay of $R C$-graphs for bigrassmannian permutations. The latter $R C$-graphs are in bijection with semistandard tableaux of rectangular shape. This is the reason for the "dispersion" remark of the introduction.

The work of [FoGrReSh97] gives a tableau rule for Schubert polynomials of a different flavor. This rule treats $\mathfrak{S}_{w}$ as a generating series for balanced fillings of the diagram of $w$. The reduction to semistandard tableaux for Grassmannian $w$ seems non-trivial.

In [BuKrTaYo04], a formula is given for a Schubert polynomial as a nonnegative integer linear combination of sum of products of Schur functions in disjoint sets of variables (with nontrivial coefficients). This is also in some sense a tableau formula for $\mathfrak{S}_{w}$. In [Le04] this result is rederived as a consequence of the crystal graph structure on $R C$-graphs developed there.

\subsection{Stable Schubert polynomials}

The stable Schubert polynomial (also known as the Stanley symmetric polynomial) is the generating series defined by

$$
F_{w}\left(x_{1}, x_{2}, \ldots\right):=\lim _{m \rightarrow \infty} \mathfrak{S}_{1^{m} \times w}
$$

where if $w \in S_{n}$ then $1^{m} \times w$ is the permutation in $S_{m+n}$ defined by

$$
\left(1^{m} \times w\right)(i)=i \text { for } 1 \leq i \leq m \text { and }\left(1^{m} \times w\right)(m+i)=m+w(i) \text { for } 1 \leq i \leq n .
$$

It is true that

$$
F_{w}\left(x_{1}, x_{2}, \ldots, x_{m}, 0,0, \ldots\right)=\mathfrak{S}_{1^{m} \times w}\left(x_{1}, \ldots, x_{m}, 0,0, \ldots\right) .
$$

Now, notice that $\lambda\left(1^{m} \times w\right)$ and $\lambda(w)$ are the same shape, but the former is shifted down $m$ steps in the grid relative to $\lambda(w)$. Therefore it follows that

$$
F_{w}\left(x_{1}, x_{2}, \ldots, x_{m}, 0,0, \ldots\right)=\sum_{T} \mathrm{wt}(T)
$$

where the sum is over all unflagged (i.e., exclude (S4)) minimal prism tableaux of shape $\lambda(w)$ that use the labels $1,2, \ldots, m$. In the limit, this argument implies the generating series $F_{w}\left(x_{1}, x_{2}, \ldots\right)$ is given by the same formula, except we allow all labels from $\mathbb{N}$. 


\subsection{An overlay interpretation of chute and ladder moves}

In [BeBi93], chute moves were defined for pipe dreams. These moves are locally of the form

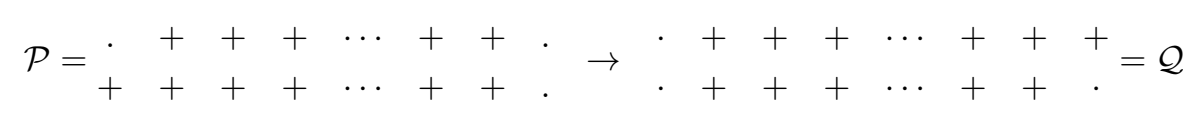

Suppose $\mathcal{P} \in \operatorname{MinPlus}\left(X_{w}^{\prime}\right), \operatorname{biGrass}(w)=\left\{u_{1}, \ldots, u_{k}\right\}$ and $\mathcal{P}=\mathcal{P}_{1} \cup \cdots \cup \mathcal{P}_{k}$, where $\mathcal{P}_{i} \in$ $\operatorname{MinPlus}\left(X_{u_{i}}^{\prime}\right)$. The following claims show:

The chute move's "long jump" of a single + may be interpreted as a sequence of the northeast local moves (3.1) applied to the $\mathcal{P}_{i}$ 's.

Example 4.1. Let $w=1432$. Now, $\operatorname{biGrass}(w)=\left\{u_{1}=1423, u_{2}=1342\right\}$. Consider the following sequence of northeast moves

$$
\left[\begin{array}{cccc}
\cdot & + & \cdot & \cdot \\
+ & ++ & \cdot & \cdot \\
\cdot & \cdot & \cdot & \cdot \\
\cdot & \cdot & \cdot & \cdot
\end{array}\right] \rightarrow\left[\begin{array}{cccc}
\cdot & + & + & \cdot \\
+ & + & \cdot & \cdot \\
\cdot & \cdot & \cdot & \cdot \\
\cdot & \cdot & \cdot & \cdot
\end{array}\right] \rightarrow\left[\begin{array}{cccc}
\cdot & ++ & + & \cdot \\
\cdot & + & \cdot & \cdot \\
\cdot & \cdot & \cdot \\
\cdot & \cdot & \cdot & \cdot
\end{array}\right]
$$

Let the support of the first and third plus diagrams be $\mathcal{P}$ and $\mathcal{Q}$, respectively. We have $\mathcal{P}, \mathcal{Q} \in \operatorname{MinPlus}\left(X_{w}^{\prime}\right)$. $\mathcal{P}$ and $\mathcal{Q}$ differ by a chute move. At the level of the overlays, one sees this transition as an application of (3.1) to each blue + in the second row.

Example 4.1 indicates the general pattern. Let $(i, j)$ be the position of the southwest + of $\mathcal{P}$ in 4.1P and $\left(i-1, j^{\prime}\right)$ the position of the northeast + of $\mathcal{Q}$ in $(4.1)$. Without loss of generality, we may assume each $\mathcal{P}_{1}, \ldots, \mathcal{P}_{t}$ contains a + at $(i, j)$ while all other $\mathcal{P}_{h}$ do not.

Claim 4.2. Consider the interval of consecutive + 's in row $i$ of $\mathcal{P}_{h}(1 \leq h \leq t)$ starting at the left with the + in position $(i, j)$. One can apply the move (3.2) (in the right to left order) to each of these + 's to obtain $\mathcal{P}_{1}^{\prime}, \ldots, \mathcal{P}_{t}^{\prime}$.

Claim 4.3. $\mathcal{P}_{h}^{\prime} \subseteq \mathcal{Q}$ for $1 \leq h \leq t$ and $\mathcal{P}_{h} \subseteq \mathcal{Q}$ for $t+1 \leq h \leq k$.

Claim 4.4. $\mathcal{Q}=\mathcal{P}_{1}^{\prime} \cup \cdots \cup \mathcal{P}_{t}^{\prime} \cup \mathcal{P}_{t+1} \cup \cdots \cup \mathcal{P}_{k}$.

A similar discussion applies to the ladder moves.

\subsection{Future work}

It is straightforward to assign weights to prism tableau in order to give a formula for double Schubert polynomials.

A generalization to Grothendieck polynomials requires a deeper control of the overlay procedure. In investigating this, one is led to some results of possibly independent interest.

Specifically, for Theorem 1.1, we have used the fact that the facets of $\Delta_{X_{w}^{\prime}}$ are intersections of facets of those associated to biGrass $(w)$. One can make a similar conjecture for all interior faces $w$ 's complex. Each $\Delta_{X_{w}^{\prime}}$ is a ball or sphere [KnMi04, Theorem 3.7]. Hence one can refer to the interior faces of this complex. Let

$$
\operatorname{IntPlus}(w)=\left\{\mathcal{P}: \mathcal{P} \in \operatorname{Plus}(w) \text { and } \mathcal{F}_{\mathcal{P}} \text { is an interior face of } \Delta_{X_{w}^{\prime}}\right\}
$$


Conjecture 4.5. $\operatorname{IntPlus}(w) \subseteq\left\{\mathcal{P}_{1} \cup \cdots \cup \mathcal{P}_{k}: \mathcal{P}_{i} \in \operatorname{IntPlus}\left(u_{i}\right)\right.$, for $\left.u_{i} \in \operatorname{biGrass}(w)\right\}$.

Conjecture 4.5 has been exhaustively computer checked for all $n \leq 6$.

As part of an intended proof of Conjecture 4.5, one defines $K$-theoretic analogues of the chute and ladder moves of [BeBi93]: that is if $\mathcal{P} \rightarrow \mathcal{Q}$ by a chute move (respectively, ladder move) then $\mathcal{P} \rightarrow \mathcal{P} \cup \mathcal{Q}$ is a $K$-chute (respectively, $K$-ladder move). Whereas not all interior plus diagrams are connected by the original chute and ladder moves, it is true that they are connected once one allows the extended moves.

The first author plans to address these and related issues elsewhere.

\section{Acknowledgements}

We thank Laura Escobar, Sergey Fomin, Victor Reiner, Steven Sam, Mark Shimozono for helpful remarks. We also made extensive use of Macaulay2 during our investigation. AY was supported by an NSF grant.

\section{References}

[BeBi93] N. Bergeron and S. Billey, RC-graphs and Schubert polynomials, Experimental Mathematics. Volume 2, Issue 4 (1993), 257-269.

[BeSo02] N. Bergeron and F. Sottile, Skew Schubert functions and the Pieri formula for flag manifolds, Trans. Amer. Math. Soc. 354 (2002), 651-673.

[BeSo98] Schubert polynomials, the Bruhat order, and the geometry of flag manifolds, Duke Math. J. 95 (1998), no. 2, 373-423.

[BiJoSt93] S. Billey, W. Jockush and R. Stanley, Some combinatorial properties of Schubert polynomials, J. of Algebraic Comb., Vol. 2, Num. 4, 1993, 345-374.

[BuKrTaYo04] A. Buch, A. Kresch, H. Tamvakis and A. Yong, Schubert Polynomials and Quiver Formulas, Duke Math. J., Vol. 122 (2004), no. 1, 125-143.

[CoTa13] O. Coşkun and M. Taşkin, Tower tableaux and Schubert polynomials, J. Combin. Theory Series A, Volume 120 Issue 8 (2013), 1976-1995.

[FoGrReSh97] S. Fomin, C. Greene, V. Reiner and M. Shimozono, Balanced labellings and Schubert polynomials, European Journal of Combinatorics 18 (1997), 373-389.

[FoKi96] S. Fomin and A. N. Kirillov, The Yang-Baxter equation, symmetric functions, and Schubert polynomials, Discrete Mathematics 153 (1996), 123-143.

[FoSt94] S. Fomin and R. P. Stanley, Schubert polynomials and the nil-Coxeter algebra, Adv. Math. 103 (1994), 196-207.

[Fu91] W. Fulton, Flags, Schubert polynomials, degeneracy loci, and determinantal formulas, Duke Math. J., Vol. 65 (1991), No. 3., 381-420.

[KaSt95] M. Kalkbrener and B. Sturmfels, Initial complexes of prime ideals, Adv. Math. 116 (1995), 365-376. 
[Kn09] A. Knutson, Frobenius splitting, point counting, and degeneration, preprint, 2009. arXiv:0911.4941

[KnMi05] A. Knutson and E. Miller, Gröbner geometry of Schubert polynomials, Annals. Math. 161 (2005), 1245-1318.

[KnMi04] _ Subword complexes in Coxeter groups, Adv. Math. 184 (2004), 161-176.

[KnMiYo09] A. Knutson, E. Miller and A. Yong, Gröbner geometry of vertex decompositions and of flagged tableaux, J. Reine Angew. Math. 630 (2009), 1-31.

[Ko00] M. Kogan, Schubert geometry of flag varieties and Gelfand-Cetlin theory, Ph.D. thesis, Massachusetts Institute of Technology, 2000.

[Ko90] A. Kohnert, Weintrauben, Polynome, Tableaux, Bayreuth Math. Schrift. 38 (1990), 1-97.

[LaSh96] A. Lascoux and M.-P. Schützenberger, Trellis et bases des groupes de Coxeter, Electron. J. Combin. 3 (1996), no. 2, R27, 35 pp. (electronic)

[LaSh85] Schubert polynomials and the Littlewood-Richardson rule, Lett. Math. Phys. 10 (1985), no. 2-3, 111-124.

[LaSh82a] _ Polynômes de Schubert, C. R. Acad. Sci. Paris Sér. I Math. 295 (1982), 629-633.

[LaSc82b]__ Structure de Hopf de l'anneau de cohomologie et de l'anneau de Grothendieck d'une variété de drapeaux, C. R. Acad. Sci. Paris, 295 (1982), 629-633.

[Le04] C. Lenart, A unified approach to combinatorial formulas for Schubert polynomials, J. Algebraic. Combin., 20 (2004), 263-299.

[Ma98] P. Magyar, Schubert polynomials and Bott-Samelson varieties, Commentarii Mathematici Helvetici 73 (1998), 603-636.

[Ma01] L. Manivel, Symmetric functions, Schubert polynomials and degeneracy loci, American Mathematical Society, Providence, RI, 2001.

[MiSt05] E. Miller and B. Sturmfels, Combinatorial Commutative Algebra, Springer Science+Business Media, Inc., 2005.

[Wi02] R. Winkel, A derivation of Kohnert's algorithm from Monk's rule, Sém. Lothar. Combin. 48 (2002), Art. B48f, 14 pp.

[Wi99] Diagram rules for the generation of Schubert polynomials, J. Combin. Th. A., 86 (1999), 14-48. 